\title{
The Feasibility and Acceptability of a Smartphone-Based Music Intervention for Acute Pain
}

\author{
Peter R Chai \\ Department of Emergency Medicine \\ Brigham and Women's Hospital \\ The Fenway Institute \\ pchai@bwh.harvard.edu
}

Guruprasad D Jambaulikar

Department of Emergency Medicine

Brigham and Women's Hospital

gjambaulikar@bwh.harvard.edu

Edward W Boyer

Department of Emergency Medicine

Brigham and Women's Hospital

The Fenway Institute

eboyer@bwh.harvard.edu

\author{
Kristin L Schreiber \\ Department of Anesthesia, \\ Perioperative, and Pain Medicine \\ Brigham and Women's Hospital \\ klschreiber@bwh.harvard.edu
}

\author{
Anna Kikut \\ Department of Emergency Medicine \\ Brigham and Women's Hospital \\ akikut@bwh.harvard.edu
}

\author{
S. Wade Taylor \\ Boston University \\ The Fenway Institute \\ wtaylor@fenwayhealth.org
}

Mohammad Adrian Hasdianda

Department of Emergency Medicine

Brigham and Women's Hospital

mhasdianda@bwh.harvard.edu

\begin{abstract}
Pain is an unpleasant experience the neurobiology of which is influenced by psychosocial factors including negative affect. Music is a ubiquitous experience that can improve affect, potentially decreasing anxiety and catastrophizing, both of which are associated with greater pain severity. We hypothesized that a machine-learning generative music intervention in the form of a smartphone web app (Unwind) could be used to modulate the experience of pain. In this pilot study, we recruited 15 individuals with acute pain who were admitted to an observation unit in the emergency department, and were being treated with opioids. Participants used the music intervention (Unwind) during this brief hospitalization, after which we assessed their response to its use through a semistructured qualitative interview. Overall, participants responded positively to Unwind. While some reported some technical challenges, participants were willing to continue using it at home. In particular, participants reported using Unwind to address their anxiety, and many used it to facilitate sleep in the presence of pain. This study demonstrates that individuals with acute pain will accept and use a smartphone-based music protocol.
\end{abstract}

\section{Introduction}

Music - the combinations of sounds organized in time - is a universal part of cultures across the world. Manipulation of different pitches within music generates melodies that evoke an emotive response. These emotive responses to music have been used across multiple cultures in human history in the form of celebration, reverence, bereavement, and healing.

The healing effect of music has been recognized since ancient times.[18] Aristotle wrote in De Anima that flute music could be used to elicit strong emotion and purify the soul. Similarly, the ancient Egyptians describe the use of musical incantations to heal the sick. In 1880, the French scientist Diogel described changes in blood pressure and heart rate in response to pleasurable music. This phenomenon, the "thrill" of listening to pleasurable music, has been demonstrated to activate dopaminergic reward pathways in the nucleus accumbens of the brain.[11,22] Additionally, the experience of listening to pleasurable music may also involve endogenous opioids in the central nervous system. The feeling of anticipation in response to selecting a pleasurable song is associated with an upregulation of endogenous circulating opioids.[17,26]

Because listening to music generates the sensation of pleasure and improves affect, researchers have considered leveraging music to decrease both acute and chronic pain. The biopsychosocial model of pain emphasizes that although the experience of pain 
arises from the biological activation of the nervous system, it is also importantly and fundamentally influenced by psychological reactions and social contexts.[10] Enhanced negative affect, anxiety, sleep disturbance, and maladaptive coping (e.g., catastrophizing) have previously been associated with greater acute or chronic pain after surgery).[9,15,24,25] Individuals who catastrophize about their pain (including aspects of rumination, magnification, and helplessness) experience greater pain severity, develop chronic pain at higher rates, and maybe at greater risk for opioid use.[8,23,24] There is some evidence that individuals with higher catastrophizing scores may in fact respond to a distracting stimulus with greater pain reduction.[23] Given that finding, it is possible that individuals with a greater element of catastrophizing or anxiety around pain may respond well to music interventions as well.

The concept of using music to address pain in the perioperative period has also been explored.[14] Investigators have demonstrated that listening to music decreases postoperative pain experienced by individuals undergoing spinal and thoracic surgery.[16,19] Similarly, individuals listening to music also used less opioids via intravenous patientcontrolled analgesia devices after surgery.[29] Music may also be efficacious in more minor surgical procedures, including burn dressing changes or bone marrow biopsies, where patients experienced less catastrophizing when they were listening to music.[7]

The development of smartphones and portable media players in the past 20 years has created novel methods in which music can be delivered to listeners. This paradigm shift of having music available in a small, mobile format creates an opportunity to generate personalized groups of songs centered around a genre (playlists), store millions of different songs for instant access on the cloud, and apply machine learning techniques to discover and generate new playlists that represent "new songs" to users. [30] The ability to generate new playlists, or entirely new songs, and measure the real-time response to music expands the potential feasibility of music as a behavioral intervention.

\subsection{Generative music in the era of cloud computing and machine learning.}

The presence of mass storage through cloud computing allows computer protocols to generate novel playlists or even novel songs based on user preferences. Currently, three major techniques of curating large libraries of music exist: personal curation, professional curation, and automated discovery (Table 1).[3]

Table 1: Music curation strategies

\begin{tabular}{|l|l|l|}
\hline Technique & Advantages & Disadvantages \\
\hline $\begin{array}{l}\text { Personal } \\
\text { Curation }\end{array}$ & $\begin{array}{l}\text { Deeply } \\
\text { personalized music } \\
\text { personal } \\
\text { knowledge of } \\
\text { songs }\end{array}$ \\
\hline $\begin{array}{l}\text { Professional } \\
\text { Curation }\end{array}$ & $\begin{array}{l}\text { Ability to listen to } \\
\text { new songs curated } \\
\text { by individuals with } \\
\text { similar musical } \\
\text { preferences }\end{array}$ & $\begin{array}{l}\text { Professionally } \\
\text { curator may not } \\
\text { have aligned } \\
\text { musical } \\
\text { preferences to } \\
\text { the user }\end{array}$ \\
\hline Automated & $\begin{array}{l}\text { Ever-new songs } \\
\text { generated through } \\
\text { machine learning } \\
\text { protocols }\end{array}$ & $\begin{array}{l}\text { Protocols may } \\
\text { need internet } \\
\text { access to utilize } \\
\text { libraries of } \\
\text { music }\end{array}$ \\
\hline
\end{tabular}

Personal and professional curation rely on humans to maintain and generate playlists of music that are either appealing to themselves or to individuals with similar musical preferences. [13,21] A user can generate their own playlist by selecting songs from a large music libraries like Apple Music, Spotify, Pandora. These playlists can be grouped by genre or activity and saved on the cloud or a mobile device for access at a later date. Similarly, professional curation is achieved by allowing professional music curators, musicians, or celebrities to create individual playlists and share them through popular music curation programs.

Automated discovery applies the fluid approach of machine learning to generate highly personalized music.[2] Automated discovery uses two techniques (collaborative filtering or content-based modeling) that when applied correctly, can theoretically generate endless music interventions that are new to the listener, yet grounded in their personal preferences.[6] Collaborative filtering uses an individual's personal musical preferences to find songs of similar style, intonation or genre, generating automated playlists (e.g., Spotify's Discover Weekly playlist) that are appealing to the user. Content-based modeling uses a machine learning protocol similar to collaborative filtering, organizing potential "pleasurable" songs into genres of intonation, rhythm and notes (e.g., musical genes). Instead of styles of music like country music or jazz, content-based modeling allows protocols to gather songs that might share features of music that the user finds appealing.

Automated discovery, combined with a machine learning protocol and user inputs, creates the 
potential to not only discover new songs that appeal to users, but also to generate new music, previously unheard and ever-new that is targeted to boost affect in individuals.[3] By identifying musical genes that alter affect through content-based modeling, a machine learning protocol can create new songs as behavioral interventions. Generative music protocols that reside on the cloud can be accessed by users through smartphones, allowing for equitable access to potential interventions as they are developed. Additionally, the highly personalized nature of generative music allows users to tailor potential interventions to their own preferences. Ideally, these music protocols would allow patient/physician dyads to work together in addressing specific disease conditions.

\subsection{Music as a behavioral intervention for pain.}

Because music boosts affect among its listeners, its use as a behavioral intervention for pain impacted by poor affect is appealing. Personalized music targeted to affect could serve as an analgesic adjunct, minimizing psychosocial contributors to pain including catastrophizing and anxiety.[10] At the same time, the biological effect of listening to music includes positive activation of endogenous dopamine transmission in reward pathways in the brain.[4,22]

In this study, we piloted the use of a personalized smartphone-based music intervention (Unwind) to address acute pain and anxiety in patients in an emergency department observation unit. We aimed to study the feasibility and acceptability of using this music intervention to address pain and anxiety in a non-emergent setting. We also sought to gather user perceptions on the strengths of the intervention and ways in which it could be deployed more effectively.

\section{Materials and Methods}

This was a prospective observational study of individuals over 18 years old who were admitted to our emergency department observation unit (ED obs) with acute pain, and who were ordered opioid analgesics by the primary emergency department team. Decisions to order opioids for potential study participants were made independently of their participation in the study. Our emergency department is an academic, urban, tertiary referral center with approximately 65,000 patient visits annually. Individuals who are admitted to the 20-bed ED observation unit are under the supervision of an emergency medicine attending physician and a trained emergency medicine physician assistant. This study was approved by the Partners Institutional Review Board (IRB). Participants were screened in the emergency department, and if they met enrollment criteria, were approached by a research assistant (RA), and verbal consent for participation was obtained. Next, basic demographic information including their history of previous opioid use was collected via a questionnaire. The RA then demonstrated the use of a novel music intervention (Unwind) housed on a smartphone's internet browser (See 2.1. The music intervention, Unwind).

Participants then demonstrated competency operating Unwind with the RA, and were instructed to use Unwind as often as they chose during the first 24 hours in ED obs.

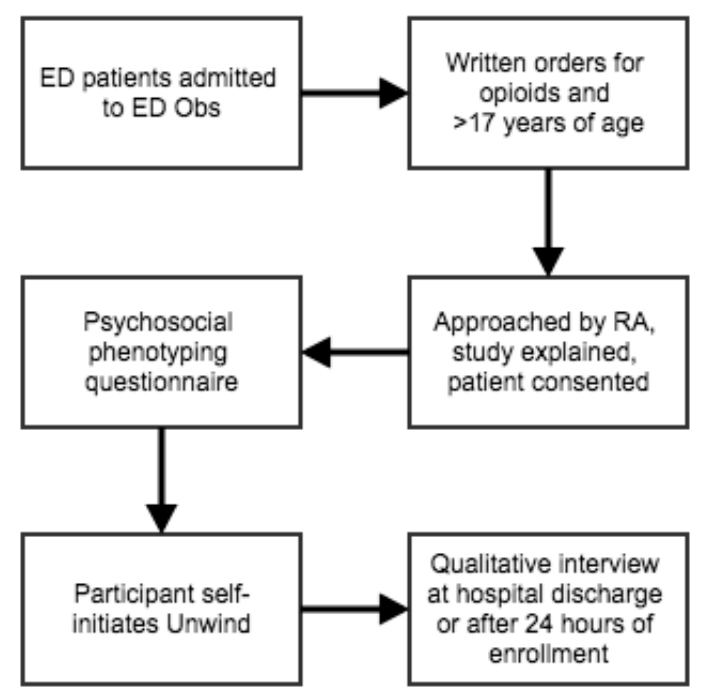

Figure 1: Study procedures

At the end of the study period, the RA collected study materials and audio recorded a semi-structured interview to help understand the use and intention to use music for pain during their hospitalization. The study team developed an interview guide grounded in the Technology Acceptance Model (TAM), to help understand the intent of participants to use Unwind, its actual use characteristics, and aspects of Unwind that could be optimized for future use.[5]

\subsection{The music intervention, Unwind}

We used a novel music web app, Unwind (Bose Corporation, Boston MA), housed on a smartphone (Apple iPhone 6) that creates music using generative music theory (Figure 2). Unwind is optimized for Safari on iOS versions 6 and greater. 


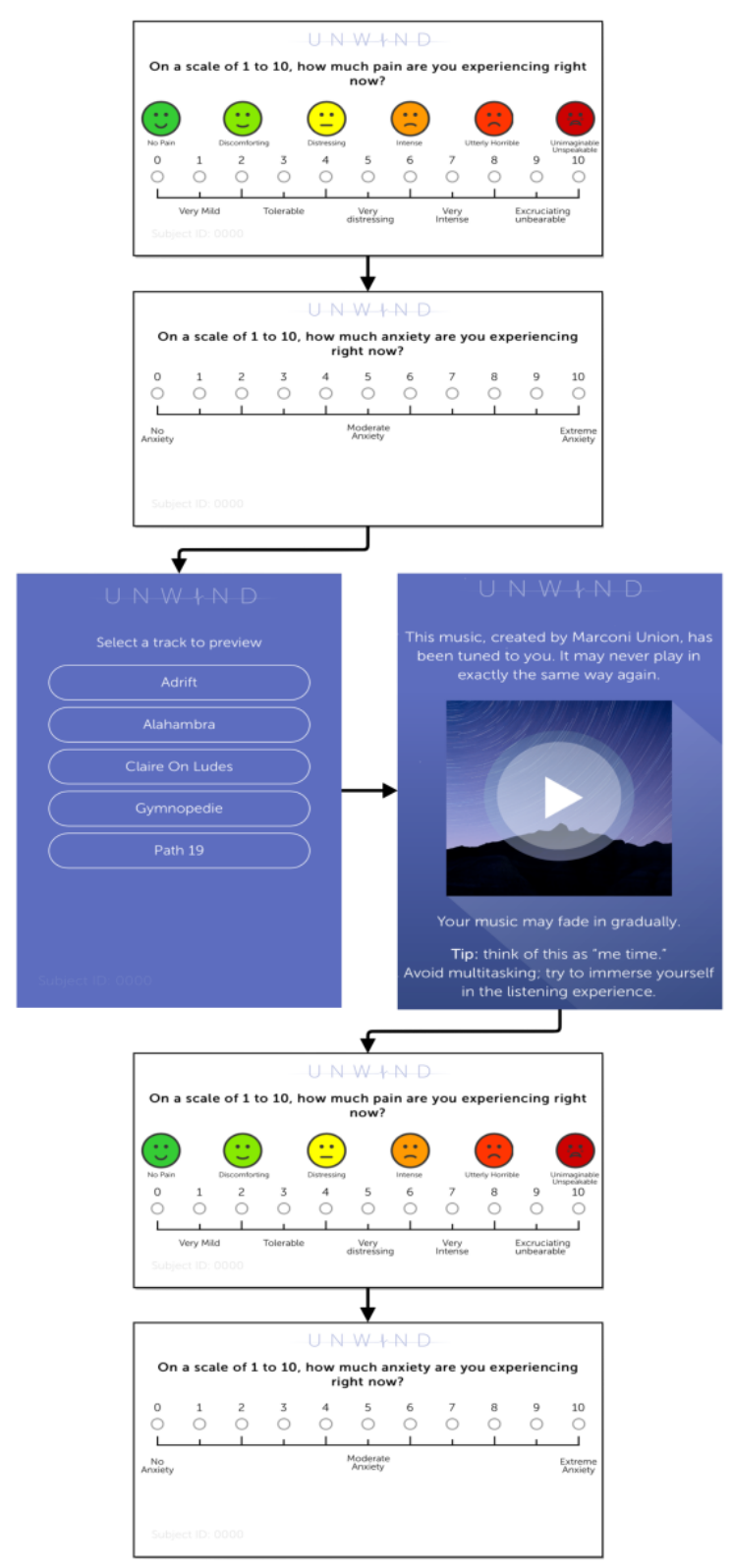

Figure 2: Process flow of Unwind

Innovatively, Unwind leverages generative music theory to create ever-new yet appealing songs using the user as composer. At its core, Unwind contains several tracks of music in isolation. For example, one track may contain several hours of drums in different rhythms and intonations, while another track contains wind instruments. The genesis of each song generated from Unwind comes from the user via a series of survey questions that ask about the level of pain and anxiety users feel, and heart rate collected using sensors on board the smartphone. Next users are presented with a library of 5 different tracks of music, and are allowed to select a track of music they find relaxing or pleasurable. Heart rate is also collected by Unwind to calibrate the initial beat. Unwind then uses data about the user's pain and anxiety to generate approximately 10 minutes of music pulling from its library of tracks. A proprietary machine algorithm trained on a dataset of known affective music generates songs so that each time Unwind is accessed, a new, yet familiar song is generated in response to changes in user-inputted pain, anxiety and collected heart rate. User preferences for the music, their average pain and anxiety scales, and the number of times they access Unwind are recorded.

\subsection{Data analysis.}

Data was collected on music utilization including the number of times participants accessed Unwind, as well as self-reported pain and anxiety before and after access to the intervention. We defined a "full completion" of Unwind as a recorded access of Unwind, and completion of both the pre-music and post-music pain/anxiety scales. A "partial completion" consisted of recorded access of Unwind, and completion of one set of pain/anxiety scales or partial completion of pain/anxiety scales.

Two authors independently listened to qualitative interviews and recorded real-time data regarding usability, acceptance, and operational features surrounding Unwind (PRC, AK). They also recorded illustrative comments from study participants verbatim. Next, we used the technique of applied thematic content analysis to understand important themes that emerged in qualitative interviews.[28] Specific codes were developed based on anticipated content areas covered in interviews. Inductive codes were added while listening to interviews. We continued coding until thematic saturation was achieved.[1] A debrief table of codes and illustrative comments from study participants was prepared and presented to the study team and reviewed for completeness. Disagreements between the initial coders were reconciled through an open discussion with the study team.

\section{Results}

During the study period, 15 participants were enrolled (Table 2). Thirty three percent $(\mathrm{N}=5)$ identified as male, and $67 \%(\mathrm{~N}=10)$ identified as female, with two individuals reporting previous opioid use. Most participants reported listening to music daily, while $40 \%(\mathrm{~N}=6)$ reported previous use of a music app like Unwind on a smartphone. All 
participants demonstrated competence in using Unwind.

Table 2: Participant demographics

\begin{tabular}{|l|l|}
\hline & $\mathrm{N}=15$ \\
\hline Mean age (yrs) & 42.5 \\
\hline Sex & \\
Male & 5 \\
Female & 10 \\
\hline Ethnicity/Race & \\
White, non-Hispanic & 8 \\
Black, non-Hispanic & 3 \\
Hispanic & 3 \\
Other & 1 \\
\hline Opioid use in past 30 & 2 \\
days & \\
\hline Music use previously & \\
Yes & 14 \\
No & 0 \\
No answer & 1 \\
\hline Previous use of music & \\
app for relaxation & 6 \\
Yes & 6 \\
No & 3 \\
No answer & \\
\hline
\end{tabular}

Participants reported that Unwind was easy to use and intuitive (Table 3). However, $46 \%(\mathrm{~N}=7)$ reported experiencing at least some challenges using Unwind. Participants reported that their challenges in using Unwind were associated with collecting through measuring heart rate on a smartphone. Despite this technical challenge, $73 \%(\mathrm{~N}=11)$ participants reported they would be willing to use Unwind or an equivalent music intervention at home and would be willing to download and use a music protocol in the form of a smartphone app. Participants used Unwind a mean of 4 times during the study period. Of these access episodes, participants fully interacted with Unwind (e.g., completed the pre-music pain/anxiety scales, listened to the music intervention, then completed the postmusic pain/anxiety scales) $26 \%$ of the time, while participants partially completed their interaction with Unwind $51 \%$ of the time. At least some of the time, the partial use of Unwind appears to be due to the participant falling asleep after beginning the session.

Table 3: Use characteristics of Unwind

\begin{tabular}{|l|l|}
\hline & $\mathrm{N}=15$ \\
\hline Easy to use & 15 \\
\hline $\begin{array}{l}\text { Technical challenges to } \\
\text { operating Unwind }\end{array}$ & 7 \\
\hline Willing to use Unwind at & 11 \\
\hline
\end{tabular}

\begin{tabular}{|l|l|}
\hline home & \\
\hline $\begin{array}{l}\text { Willing to access } \\
\text { Unwind as a smartphone } \\
\text { app }\end{array}$ & 12 \\
\hline Technology use characteristics \\
\hline $\begin{array}{l}\text { Confirmed instances of } \\
\text { Unwind use }\end{array}$ & 57 \\
\hline $\begin{array}{l}\text { Full completion of pre } \\
\text { and post assessment }\end{array}$ & 15 \\
\hline $\begin{array}{l}\text { Partial completion of pre } \\
\text { and post assessment }\end{array}$ & 42 \\
\hline
\end{tabular}

Forty six percent $(\mathrm{N}=7)$ participants reported that they used Unwind to address anxiety provoked by their acute pain, while one participant specifically used Unwind to sleep. The other seven participants mentioned that Unwind was both relaxing and aiding their sleep. Thematically, two major drivers of Unwind use emerged from the summative comments from participants: music as anxiolysis and music as distraction (Table 4). We also identified individuals who described the integration of Unwind with other wearable devices, which may inform further automation of this intervention in the future.

Table 4: Illustrative comments from study participants on using Unwind

\begin{tabular}{|c|c|}
\hline Theme & Comment \\
\hline Anxiolysis & $\begin{array}{l}\text { "If I was looking for something to } \\
\text { calm my anxiety, I would use this } \\
\text { music." } \\
\text { "Music is a good way to remove the } \\
\text { anxiety. Every time I used it, I fell } \\
\text { asleep." }\end{array}$ \\
\hline Distraction & $\begin{array}{l}\text { "Pain is intensified when you're really } \\
\text { thinking of it. Unwind helped me } \\
\text { unfocus from pain, and it felt like not a } \\
\text { big issue." } \\
\text { "To relieve pain, you have to focus } \\
\text { really hard on the music." }\end{array}$ \\
\hline $\begin{array}{l}\text { Wearable } \\
\text { integration }\end{array}$ & $\begin{array}{l}\text { "I would like it to be linked to another } \\
\text { device so that it would be able to } \\
\text { automatically start or stop the music } \\
\text { based on how I was feeling." } \\
\text { "I liked how the phone collected my } \\
\text { heart rate. It felt like I was connecting } \\
\text { to the music." }\end{array}$ \\
\hline $\begin{array}{l}\text { Music } \\
\text { choice }\end{array}$ & $\begin{array}{l}\text { "I would have preferred different types } \\
\text { of music. It sounded like elevator } \\
\text { music." }\end{array}$ \\
\hline
\end{tabular}


"The music was really soothing. I fell asleep a couple of times."

\section{Discussion}

This pilot study provides preliminary evidence that a personalized, machine-learning generative music intervention is feasible and acceptable among individuals who report acute pain in an ED setting. Participants interacted with the music intervention despite experiencing pain, and answered brief questions regarding their pain and anxiety in realtime. Our qualitative analysis of post-use interviews found that individuals used the music intervention to decrease anxiety and as a distraction to help them cope with pain. These results suggest that music may counter psychosocial influencers of pain including anxiety and catastrophizing.

Another emerging qualitative theme was that participants thought the music intervention distracted them from pain. Pain catastrophizing, which includes an aspect of rumination about pain, has been shown to increase pain severity ratings.[12,27] Distraction has previously proven to reduce pain amongst higher catastrophizing individuals with chronic pain.[23] Further research could investigate whether a decrease in various aspects of catastrophizing about pain, or a reduction in anxiety, may mediate music's effect in reducing pain severity. Behavioral therapies to reduce pain such as cognitive behavioral therapy, may also reduce pain catastrophizing [20], and, while effective, require a significant amount of behavioral activation and training. This phone-based music intervention is less resource intensive, and may be used in the absence, or serve as an addition to behavioral programs that decrease catastrophizing and anxiety about pain. Our qualitative analysis indicated that most of the participants in this investigation described using Unwind to decrease their anxiety surrounding pain, suggesting that this innovative music intervention may decrease anxiety and catastrophizing about pain, with a potential for simultaneously decreasing related opioid use.

Regarding the feasibility of use of this intervention, we observed that individuals experiencing acute pain found it acceptable to access a music interaction, interact with simple commands and answer questions regarding their pain and anxiety in real-time. Our study participants accessed Unwind an average of four times during their involvement in the study. All participants at least partially interacted with Unwind, demonstrating that we are able to collect real-time data regarding pain and anxiety in individuals interacting with a smartphone-based music intervention while experiencing acute pain. This is significant because it suggests that future iterations of our music intervention can include brief, ecological momentary assessment (EMA) which will increase ecological validity pain measurement. Additionally, this finding also creates the opportunity to develop brief behavioral interventions that address pain in the context of a music intervention.

One important advantage to Unwind is its highly personal accessible nature. After a brief training by our research team, participants were able to use Unwind by themselves as they experienced pain. Compared to other interventions for pain where successful use may require a trained therapist, nurse or other staff member to continually administer the intervention, music is unique because it does not require ancillary staff to deliver the intervention.

\subsection{Recommendations for Future Research and Practice}

While Unwind is an acceptable and feasible music protocol that addresses acute pain, iterative improvements that improve its usability are still required. This investigation describes specific content areas of Unwind that may be targeted for improvement - collection of biometric data, improved assessment of pain and circumstances surrounding music use, and increased variation of music interventions. Iterative improvements in the user interface, include packaging the web app into a smartphone app may improve its acceptability, and allow for more rigorous access metrics to be measured. Additionally, the types of music that are offered to patients and the context in which music interventions integrate into the continuum of pain management will need to be explored.

Some participants described a desire to integrate better personalized biometric monitoring to help calibrate Unwind. We anticipate future iterations of Unwind will have the option of collecting sleep data or heart rate from a wearable device to further tailor delivered music interventions.

\subsection{Implications for Health Equity}

We continue to struggle to provide the best, equitable care to all patients. Pain is an experience that impacts all humans, yet access to innovative strategies that address pain may be limited to those with connections to academic medical centers or research institutions. Even there, the cost of developing and evaluating novel interventions for 
pain limits access to some individuals who may need these therapies the most.

The ubiquitous nature of music provides an opportunity to develop interventions that address pain that are accessible for all individuals. Music interventions, if found to be efficacious for specific painful conditions, are highly scalable through an individual's smartphone. Music that is easily accessible provides the opportunity for individuals who may need nonpharmacological interventions to use them with minimal infrastructure. Barriers like transportation and housing may traditionally impact an individual's ability to make appointments with medical providers and access behavioral health; music interventions administered via smartphone and tailored to the individual experience of pain can be a first step towards improving health equity with regards to pain.

\subsection{Limitations}

There are several limitations to this study. First, the region in which we practice and enrolled participants has been especially impacted by the opioid epidemic in the United States. Public education regarding adverse events related to opioids and nonmedical opioid use may have increased the acceptability of the music intervention as an alternative to treat acute pain. Second, the music intervention is housed on a smartphone which requires constant internet access in order to generate songs. The feasibility of such an intervention may be impacted in different populations without access to smartphone technology or with poor wireless internet access. Third, participants we recruited for the study were proficient in operating smartphones. Individuals with acute pain who have difficulty operating a smartphone may need a music intervention delivered in an alternative manner. Fourth, the study environment, an emergency department observation unit, is a highly supervised inpatient hospital setting. The use of the music intervention may differ when deployed in the outpatient setting.

\section{Conclusion}

This personalized, machine-learning generative music intervention is acceptable and feasible for use in hospitalized patients, and qualitatively decreased pain in these individuals, possibly through distraction and anxiety reduction. These findings will inform and support future investigations into the efficacy, scalability, and mechanistic underpinnings machine generated music to provide adjunctive analgesia. Wide accessibility of music interventions through smartphones provides the opportunity to improve health equity in pain.

\section{Funding sources}

This investigation was supported by NIH K24DA037109 (PI: Boyer). Dr. Schreiber is supported by NIH K23GM110540, Dr. Chai is supported by NIH K23DA044874.

\section{References}

[1] Ando, H., Cousins, R., and Young, C. Achieving Saturation in Thematic Analysis: Development and Refinement of a Codebook. Comprehensive Psychology 3, (2014), 03.CP.3.4.

[2] Bonnin, G. and Jannach, D. Automated Generation of Music Playlists: Survey and Experiments. ACM Computing Surveys 47, 2 (2015), $1-35$.

[3] Chai, P.R., Carreiro, S., Ranney, M.L., et al. Music as an Adjunct to Opioid-Based Analgesia. Journal of Medical Toxicology 64, (2017), 1378.

[4] Chanda, M.L. and Levitin, D.J. The neurochemistry of music. Trends in cognitive sciences 17, 4 (2013), 179-193.

[5] Chuttur, M. Overview of the Technology Acceptance Model: Origins, Developments and Future Directions. Sprouts Working Papers on Information Systems 9, 37 (2009), 1-23.

[6] Dannenberg, R.B. and Hu, N. Pattern Discovery Techniques for Music Audio. Journal of New Music Research 32, 2 (2003), 153-163.

[7] Eckhouse, D.R., Hurd, M., Cotter-Schaufele, S., Sulo, S., Sokolowski, M., and Barbour, L. A randomized controlled trial to determine the effects of music and relaxation interventions on perceived anxiety in hospitalized patients receiving orthopaedic or cancer treatment. Orthopedic nursing 33, 6 (2014), $342-351$.

[8] Edwards, R.R., Cahalan, C., Calahan, C., Mensing, G., Smith, M., and Haythornthwaite, J.A. Pain, catastrophizing, and depression in the rheumatic diseases. Nature reviews. Rheumatology 7 , 4 (2011), 216-224.

[9] Edwards, R.R., Mensing, G., Cahalan, C., et al. 
Alteration in pain modulation in women with persistent pain after lumpectomy: influence of catastrophizing. Journal of pain and symptom management 46, 1 (2013), 30-42.

[10] Gatchel, R.J., Peng, Y.B., Peters, M.L., Fuchs, P.N., and Turk, D.C. The biopsychosocial approach to chronic pain: scientific advances and future directions. Psychological Bulletin 133, 4 (2007), 581-624.

[11] Goldstein, A. Thrills in response to music and other stimuli. Physiological Psychology 8, 1 (1980), 126-129.

[12] Granot, M. and Ferber, S.G. The roles of pain catastrophizing and anxiety in the prediction of postoperative pain intensity: a prospective study. Clin J Pain 21, 5 (2005), 439-445.

[13] Hagen, A.N. The Playlist Experience: Personal Playlists in Music Streaming Services. Popular Music and Society 38, 5 (2015), 625-645.

[14] Hole, J., Hirsch, M., Ball, E., and Meads, C. Music as an aid for postoperative recovery in adults: a systematic review and meta-analysis. Lancet (London, England) 386, 10004 (2015), 1659-1671.

[15] Katz, J., Poleshuck, E.L., Andrus, C.H., et al. Risk factors for acute pain and its persistence following breast cancer surgery. Pain 119, 1-3 (2005), 16-25.

[16] Liu, Y. and Petrini, M.A. Effects of music therapy on pain, anxiety, and vital signs in patients after thoracic surgery. Complementary therapies in medicine 23, 5 (2015), 714-718.

[17] Mallik, A., Chanda, M.L., and Levitin, D.J. Anhedonia to music and mu-opioids: Evidence from the administration of naltrexone. Scientific reports 7 , (2017), 41952.

[18] Meymandi, A. Music, medicine, healing, and the genome project. Psychiatry (Edgmont (Pa. :

Township)) 6, 9 (2009), 43-45.

[19] Mondanaro, J.F., Homel, P., Lonner, B., Shepp, J., Lichtensztein, M., and Loewy, J.V. Music Therapy Increases Comfort and Reduces Pain in Patients Recovering From Spine Surgery. American journal of orthopedics (Belle Mead, N.J.) 46, 1 (2017), E13E22.
[20] Morley, S., Eccleston, C., and Williams, A. Systematic review and meta-analysis of randomized controlled trials of cognitive behaviour therapy and behaviour therapy for chronic pain in adults, excluding headache. Pain 80, 1-2 (1999), 1-13.

[21] Morris, J.W. and Powers, D. Control, curation and musical experience in streaming music services. Creative Industries Journal 8, 2 (2015), 106-122.

[22] Salimpoor, V.N., Benovoy, M., Larcher, K., Dagher, A., and Zatorre, R.J. Anatomically distinct dopamine release during anticipation and experience of peak emotion to music. Nature Neuroscience 14, 2 (2011), 257-262.

[23] Schreiber, K.L., Campbell, C., Martel, M.O., et al. Distraction analgesia in chronic pain patients: the impact of catastrophizing. Anesthesiology 121, 6 (2014), 1292-1301.

[24] Schreiber, K.L., Kehlet, H., Belfer, I., and Edwards, R.R. Predicting, preventing and managing persistent pain after breast cancer surgery: the importance of psychosocial factors. Pain Manag 4, 6 (2014), 445-459.

[25] Sobol-Kwapinska, M., Bąbel, P., Plotek, W., and Stelcer, B. Psychological correlates of acute postsurgical pain: A systematic review and metaanalysis. European journal of pain (London, England) 20, 10 (2016), 1573-1586.

[26] Stefano, G.B., Zhu, W., Cadet, P., Salamon, E., and Mantione, K.J. Music alters constitutively expressed opiate and cytokine processes in listeners. Medical science monitor : international medical journal of experimental and clinical research 10, 6 (2004), MS18-27.

[27] Sullivan, M.J., Thorn, B., Haythornthwaite, J.A., et al. Theoretical perspectives on the relation between catastrophizing and pain. Clin J Pain 17, 1 (2001), 52-64.

[28] Vaismoradi, M., Turunen, H., and Bondas, T. Content analysis and thematic analysis: Implications for conducting a qualitative descriptive study. Nursing \& health sciences 15, 3 (2013), 398-405.

[29] Wang, Y., Tang, H., Guo, Q., et al. Effects of Intravenous Patient-Controlled Sufentanil Analgesia and Music Therapy on Pain and Hemodynamics After Surgery for Lung Cancer: A Randomized Parallel Study. Journal of alternative and 
complementary medicine (New York, N.Y.) 21, 11 (2015), 667-672.

[30] Wikström, P. and DeFillippi, R. Streaming music services and the future of music. Edward Elgar Publishing. 